Ophthalmologica 1953;126:I-IV

\title{
Contents, Vol. 126, 1953
}

\section{Index}

Agatwal, L. P., and /. N. Raízada, The Role of Pyrazole Derivatives (Irgapyrin-Geigy) in the Treatment of Ocular Conditions 110

Alberth, B., Tränensacktuberkulose und Dakryocystorhinostomie 168

Amsler, Marc, Zur Frage des pterygiumbedingten Astigmatismus . 52

Balavoine, C, vide C. A. Baud.

Bastenie, P., vide P. Danis.

Baud, C.A., et C. Balavoine, L'ultrastructure de la membrane de

Descemet et de ses derives pathologiques (stries hyalines) . 290

Berger, G. P. von, vide Marcel Monnier.

Bouman, M.A., and J. tenDoesschate, Nervous and Photochemical

Components in Visual Adaptation 222

Busacca, Archimède, Un nouveau phénomène observe dans le

corps vitré antérieur au cours des uvéites 355

Danis, P., et P. Bastenie, Les atteintes du nerf optique au cours des

exophtalmies ædémateuses endocriniennes 65

Deweer, J. P., vide J. Francois.

Doesschate, J. ten, Observations on the Entoptic Foveal Chagrin . 148

- , vide M. A. Bouman.

Ennema, M. C, and W. P. C. Zeeman, Venous Occlusions in the

Retina 329

Fazakas, Alexander, Zusammenfassender Bericht über meine oph-

thalmologischen Pilzuntersuchungen 91

- Orbitalplastik nach Kriegsverletzungen 157

Francois, J., et J. P. Deweer, Albinisme oculaire lie au sexe et alterations caractéristiques du fond d'æil chez les femmes hétéro-zygotes (avec Planche I) 209

Francois, J., M. Rabaey et G. van der Meerssche, L'ultrastructure des tissus oculaires au

microscope électronique. I. Etude des grains pigmentaires de Гépithélium rétinien .... 347

Friede, Reínhard, Zur Operation und Prophylaxe des echten

Pterygium 161

- Über sclero-keratoplastische Eingriffe bei Geschwülsten desAugapfels 295

Glees, M., Beiderseitiger Mikrophthalmus bei nachgewiesener kon-nataler Toxoplasmose.

Klinische und histologische Beobach-

tungen 361

Ibrahim, M. G., vide H. Puxkandl.

Jonkers, G. H., Intra-Ocular Acrylic Lenses. A case report . 55 Kaeser, E. H., Das arteriovenöse Aneurysma im Sinus cavernosus 257 Kluyskens, J., et J. Titeca, Examen Electro-

encéphalographique

du Champ Visuel 
Koch, C, Über Glaskörperfäden. Morphologisch-experimentelle

Beobachtungen am Glaskörper des Thunfisches . 58

Konstas, Konstantin A., Beitrag z. Studium der Cataracta centralis 231 Krümmel, H., Epitheloidzellige Granulomatose (Boecksches Sar-

koid) an der Bindehaut 193

Ljustina-Ivancic, Vevenka, Tetra-Äthyl-Ammonium in der Thera-

pie des Glaucoms 378

Malbrán, Jorge L., José M. Paunessa, and Flaminio Vidal, Hereditary Crystalline Degeneration of the Cornea .... 369

Marzio, Q. Di, and A. Strazzi, X-ray Treatment in Uveitis . . 1

Meerssche, G., van der, vide J. Francois.

Monnier, Marcel, et G. P. von Berger, Analyse des ripostes élec-triques du centre visuel cortical

à la stimulation lumineusechez Thomme 15

Morawiecki, J., Behandlung der skrofulösen Augenentzündung

mit Privin-Cibazol-Emulsion 122

Morone, G., vide L. Venco.

Nover, Arno, Beitrag zur pathologischen Analomie der malignen

Orbitaltumoren 35

Orbán, T., vide M. Radnót.

Pajtás, J., Zur Heilung der Periphlebitis tuberculosa mittels Elek-

trokoagulation, mit Streptomycin und PAS kombiniert . . 116

Paunessa, José M., vide Jorge L. Malbrán.

Pavisic, Zvonimir, Operative Behandlung des trachomatösen Sym-

blepharons 240

Puxkandl, H., M. G. Ibrahim und H. Tewfik, Untersuchungenüber die Diffusion von

Isonikotinsäurehydrazid in das Kam-merwasser 387

Rabaey, M., vide J. Francois.

Radnót, M., und T. Orbán, Über experimentell erzeugte dauemde

Tensionserhöhung beim Kaninchen 245

Raizada, I. N, vide L. P. Agarwal.

Rama, G., Tumeur pigmentée de la loge lacrymale ... 44

Strazzi, A., vide Q. Di Marzio.

Szekler, Robert, Ein Uvealsarkom bei einem Mitglied einer Fa-

milie mit Recklinghausenscher Krankheit 248

Tewfik, H., vide H. Puxkandl.

Titeca, J., vide /. Kluyskens.

Venco, L., et G. Morone, Manifestations pupillaires dans les contusions du globe oculaire 282

Vidal, Flaminio, vide Jorge L. Malbrán.

Zeeman, W. P. C., vide M. C. Ennema.

GESELLSCHAFTSBERICHTE - SOCIETY TRANSACTIONS - SOCIÉTÉS

The Netherlands Ophthalmological Society

125th Meeting, Leyden, May Srd and 4th, 1952172

126th Meeting, Groningen, September 20th and 21st, 1952 .... 308

NECROLOGIA

Karl Wessely, 1874-1953 252

BUCHBESPRECHUNGEN - BOOK REVIEWS - LIVRE 5/8 NOUVEAUX 
6. 64

126

189

253

326

393

VARIA 64

256

396 